\title{
On semi-transitive orientability of Kneser graphs and their complements
}

\author{
Sergey Kitaev* and Akira Saito ${ }^{\dagger}$
}

March 8, 2019

\begin{abstract}
An orientation of a graph is semi-transitive if it is acyclic, and for any directed path $v_{0} \rightarrow v_{1} \rightarrow \cdots \rightarrow v_{k}$ either there is no edge between $v_{0}$ and $v_{k}$, or $v_{i} \rightarrow v_{j}$ is an edge for all $0 \leq i<j \leq k$. An undirected graph is semi-transitive if it admits a semi-transitive orientation. Semitransitive graphs include several important classes of graphs such as 3-colorable graphs, comparability graphs, and circle graphs, and they are precisely the class of word-representable graphs studied extensively in the literature.

In this paper, we study semi-transitive orientability of the celebrated Kneser graph $K(n, k)$, which is the graph whose vertices correspond to the $k$-element subsets of a set of $n$ elements, and where two vertices are adjacent if and only if the two corresponding sets are disjoint. We show that for $n \geq 15 k-24, K(n, k)$ is not semitransitive, while for $k \leq n \leq 2 k+1, K(n, k)$ is semi-transitive. Also, we show computationally that a subgraph $S$ on 16 vertices and 36 edges of $K(8,3)$, and thus $K(8,3)$ itself on 56 vertices and 280 edges, is non-semi-transitive. $S$ and $K(8,3)$ are the first explicit examples of triangle-free non-semi-transitive graphs, whose existence was established via Erdős' theorem by Halldórsson et al. in 2011. Moreover, we show that the complement graph $\overline{K(n, k)}$ of $K(n, k)$ is semi-transitive if and only if $n \geq 2 k$.
\end{abstract}

\footnotetext{
${ }^{*}$ Department of Computer and Information Sciences, University of Strathclyde, 26 Richmond Street, Glasgow G1, 1XH, United Kingdom. Email: sergey.kitaev@cis.strath.ac.uk.

${ }^{\dagger}$ Department of Information Science, Nihon University, Sakurajosui 3-25-40 SetagayaKu Tokyo 156-8550, Japan. Email: asaito@chs.nihon-u.ac.jp.
} 


\section{Introduction}

An orientation of a graph is semi-transitive if it is acyclic, and for any directed path $v_{0} \rightarrow v_{1} \rightarrow \cdots \rightarrow v_{k}$ either there is no edge between $v_{0}$ and $v_{k}$, or $v_{i} \rightarrow v_{j}$ is an edge for all $0 \leq i<j \leq k$. The notion of a semi-transitive orientation generalizes that of a transitive orientation. An undirected graph is semi-transitive if it admits a semi-transitive orientation. Not all graphs are semi-transitive, and the minimum (by the number of vertices) non-semitransitive graph is the wheel graph $W_{5}$ on 6 vertices. Note that any complete graph can be oriented transitively, and thus semi-transitively.

A shortcut $C$ in a directed acyclic graph is an induced subgraph on vertices $\left\{v_{0}, v_{1}, \ldots, v_{k}\right\}$ for $k \geq 4$ such that $v_{0} \rightarrow v_{1} \rightarrow \cdots \rightarrow v_{k}$ is a directed path, $v_{0} \rightarrow v_{k}$ is an edge, and there exist $0 \leq i<j \leq k$ such that there is no edge between $v_{i}$ and $v_{j}$. Thus, $C$ has no directed cycles and it is non-transitive, and an orientation is semi-transitive if and only if it is acyclic and shortcut-free. The edge $v_{0} \rightarrow v_{k}$ in $C$ is called the shortcutting edge, and the path $v_{0} \rightarrow v_{1} \rightarrow \cdots \rightarrow v_{k}$ is the long path in $C$.

The notion of a semi-transitive orientation was introduced by Halldórsson, Kitaev and Pyatkin [6] in 2011 as a powerful tool to study word-representable graphs defined in Section 2 via alternation of letters in words and studied extensively in the recent years (see [8, 9] and references therein). The class of semi-transitive graphs is precisely the hereditary class of word-representable graphs. The roots of the theory of word-representable graphs, i.e. semitransitive graphs, are in the study of the celebrated Perkins semigroup in [1], which has played a central role in semigroup theory since 1960, particularly as a source of examples and counterexamples. However, the significance of the class of semi-transitive graphs is in the fact that it includes several important classes of graphs such as 3-colorable graphs, comparability graphs and circle graphs [8, 9]. Note that the user-friendly software [5] by Glen is of special importance for the development of the area, and we use it in this paper to show a particular result.

For any two integers $k \geq 1$ and $n \geq k$, the Kneser graph $K(n, k)$ is the graph whose vertices correspond to the $k$-element subsets of a set $[n]:=\{1,2, \ldots, n\}$, where two vertices are adjacent if and only if the two corresponding sets are disjoint 1 . In particular, $K(5,2)$ is isomorphic to the celebrated Petersen graph. When writing down subsets, we omit brackets and commas. Thus, for example, the subset $\{1,4,6,7\}$ is recorded by us

\footnotetext{
${ }^{1}$ Often, when defining the Kneser graph $K(n, k)$, the assumption is that $n \geq 2 k+1$ to avoid dealing with null graphs, which are meaningful in our context, since we also deal with $\overline{K(n, k)}$.
} 
as 1467 , while the subset $\{2,9,10\}$ as $29(10)$, etc. A Kneser graph is vertex transitive and edge transitive, and these graphs are named after Martin Kneser, who first investigated them in 1955. There is a long line of research dedicated to Kneser graphs; see the recent [13] and references therein.

In this paper we study semi-transitive orientability of Kneser graphs and their complements. Our main results can be summarized as follows:

- For $n \geq 15 k-24, K(n, k)$ is not semi-transitive (see Theorem[13), while for $k \leq n \leq 2 k+1 K(n, k)$ is semi-transitive (see Theorem 9). Moreover, it is shown by the software [5] that $K(8,3)$ is not semi-transitive (see the discussion at the end of Section 3 ). A certain subgraph $S$ of $K(8,3)$ presented in Figure 1 and $K(8,3)$ itself are the first explicit examples of triangle-free non-semi-transitive graphs, whose existence was established via Erdős' theorem by Halldórsson, Kitaev and Pyatkin in [6].

- The complement graph $\overline{K(n, k)}$ of $K(n, k)$ is semi-transitive if and only if $n \geq 2 k$ (see Theorem [18).

\section{Known results to be used in the paper}

We begin with the following well known result.

Theorem 1. Let $G$ be an acyclically oriented graph with chromatic number $m$. Then, $G$ contains a directed path of length at least $m-1$.

Proof. Any acyclic orientation contains a source, i.e. a vertex with no incoming edges. Consider all sources and colour them in colour 1. Remove all sources along with the edges connected to them, and colour the sources in the obtained acyclic graph in colour 2. Proceed in this way. If the longest path in $G$ is of length at most $m-2$, then it is possible to colour $G$ in at most $m-1$ colours contradicting the chromatic number of $G$ being $m$. Thus, $G$ contains a directed path of length at least $m-1$.

\subsection{Kneser graphs and their complements}

Theorem 2 ([12]). For $n \geq 2 k-1$, the chromatic number of the Kneser graph $K(n, k)$ is $n-2 k+2$.

The following is a well known and easy to see fact.

Lemma 3. When $n<c k, K(n, k)$ does not contain cliques $K_{c}$ of size $c$, whereas it does contain such cliques when $n \geq c k$. 
An independent set is a set of vertices no two of which are connected by an edge. The independence number of a graph is the size of a maximal independent set.

Theorem 4 (The Erdös-Ko-Rado Theorem; 4]). The independence number of the Kneser graph $K(n, k)$, equivalently, the size of the largest clique in $\overline{K(n, k)}$, is $\left(\begin{array}{l}n-1 \\ k-1\end{array}\right)$.

Theorem 5 ([2]). The chromatic number of the graph $\overline{K(n, k)}$ is $\left\lceil\left(\begin{array}{l}n \\ k\end{array}\right) /\left\lfloor\frac{n}{k}\right\rfloor\right\rceil$.

\subsection{Semi-transitive graphs and word-representability}

Theorem 6 ([7]). Any 3-colourable graph is semi-transitive.

Proof. Colour the vertices in a given 3-colourable graph $G$ in colours 1, 2 and 3, and orient the edges from a smaller colour to a larger colour. Such orientation is clearly acyclic. Moreover, it is shortcut-free since the longest direct path is of length 2 , while for a shortcut we need a directed path of length 3 . Thus, the orientation is semi-transitive.

Lemma 7 ([1]). Suppose that the vertices in $\{a, b, c, d\}$ induce a subgraph $S$ in a partially oriented graph such that $a \rightarrow b$ and $b \rightarrow c$ are edges, $c d$ and da are non-oriented edges, and $S$ is different from the complete graph $K_{4}$. Then, the unique way to orient $c d$ and da in order not to create a directed cycle or a shortcut is $a \rightarrow d$ and $d \rightarrow c$.

Proof. Indeed, suppose that the edge $c d$ is oriented as $c \rightarrow d$. Then, orienting $a d$ will either give the cycle $a \rightarrow b \rightarrow c \rightarrow d \rightarrow a$, or the shortcut with the shortcutting edge $a \rightarrow d$. Thus, the orientation of $c d$ must be $d \rightarrow c$. To complete the proof, we note that orienting $d a$ as $d \rightarrow a$ will give a shortcut with the shortcutting edge $d \rightarrow c$.

To accomodate a simple proof of Theorem 15, next we introduce the notion of a word-representable graph and state the relation between semitransitive graphs and word-representable graphs in Theorem 8.

Letters $x$ and $y$ alternate in a word $w$ if after deleting in $w$ all letters but the copies of $x$ and $y$ we either obtain a word $x y x y \cdots$ (of even or odd length) or a word yxyx... (of even or odd length). For example, the letters 2 and 5 alternate in the word 11245431252, while the letters 2 and 4 do not alternate in this word. A simple graph $G=(V, E)$ is word-representable if there exists a word $w$ over the alphabet $V$ such that letters $x$ and $y$ alternate in $w$ iff $x y \in E$. By definition, $w$ must contain each letter in $V$. We say that 
$w$ represents $G$. For example, each complete graph $K_{n}$ can be represented by any permutation $\pi$ of $\{1,2, \ldots, n\}$, or by $\pi$ concatenated any number of times. Also, the empty graph $E_{n}$ (also known as an edgeless graph, or a null graph) on vertices $\{1,2, \ldots, n\}$ can be represented by $1122 \cdots n n$, or by any permutation concatenated with the same permutation written in the reverse order.

Theorem 8 ([7]). A graph is semi-transitive if and only if it is wordrepresentable.

\section{Semi-transitivity of Kneser graphs}

Theorem 9. For $k \leq n \leq 2 k+1, K(n, k)$ is semi-transitive.

Proof. By Theorem 2, for $n \leq 2 k+1, K(n, k)$ is 3-colourable, and thus is semi-transitive by Theorem 6 ,

The following lemma is easy to see.

Lemma 10. The graph $K(n, k)$ (resp., $\overline{K(n, k)})$ is an induced subgraph in any graph $K(m, k)$ (resp., $\overline{K(m, k)})$ for $m \geq n$.

Proof. The subgraph of $K(m, k)$ induced only by the vertices formed by the elements in $\{1,2, \ldots, n\}$ is isomorphic to $K(n, k)$. The statement for the complements now follows as well.

In the following theorem, we repeatedly use the fact that $K(6,2)$ is $K_{4}$ free by Lemma 3, which allows the application of Lemma 7.

Theorem 11. The Kneser graph $K(n, 2)$ is not semi-transitive for $n \geq 6$.

Proof. By Lemma 10, and the hereditary nature of semi-transitivity, it is sufficient to prove the theorem for $K(6,2)$.

We proceed by contradiction. Assume that $K(6,2)$ can be oriented semitransitively and fix such an orientation. Since the chromatic number of $K(6,2)$ is 4 by Theorem 2 , by Theorem 1 the oriented copy of $K(6,2)$ must contain a directed path $A \rightarrow B \rightarrow C \rightarrow D$. Note that if the edge $A \rightarrow D$ exists, we would obtain a contradiction, since the vertices $A, B, C, D$ would induce a shortcut $\left(K(6,2)\right.$ is $K_{4}$-free). Thus, there is no edge in $K(6,2)$ between $A$ and $D$, and without loss of generality, we can assume that one of the following three cases occurs, where abcdef is a permutation of [6]: 
Case 1. $A=a b, B=c d, C=b e$ and $D=a f$ ( $C$ is uniquely determined once the assumption that $D$ involves $a$ is made). Note that in this case, $B \rightarrow D$ is an edge.

Case 2. $A=a b, B=c d, C=e f$ and $D=a c$ (the assumption here is that $D$ shares an element with $A$ and $B$ ). This case is equivalent to Case 1, since reversing all edges in a semi-transitive orientation gives a semitransitive orientation, and the letters $a, b, \ldots$ can be renamed. So, Case 2 does not need to be considered.

Case 3. $A=a b, B=c d, C=b e$ and $D=a c$ (the assumption here is that $D$ shares an element with $A$ and $B$, and $C$ shares an element with $A)$. In this case, consider the 4-cycle induced by $A, B, C$ and $d f$. By Lemma 7, we must have the following edges: $A \rightarrow d f$ and $d f \rightarrow C$. But then, the directed path $A \rightarrow d f \rightarrow C \rightarrow D$ is equivalent to the path in Case 1, so Case 3 does not need to be considered.

Thus, we only need to consider Case 1 and arrive at a contradiction. Our strategy here is to consider a number of graphs induced by 4 vertices (in certain order) in which orientation of edges is uniquely determined from our assumptions. Eventually, we will show that shortcuts are unavoidable. In what follows, for convenience, we do not use the letters $A, B, C$ and $D$, writing the 2 -set partitions corresponding to them instead.

- From the graph induced by $a b, d f, c d$, be, by Lemma 7, we must have $a b \rightarrow d f$ and $d f \rightarrow b e$.

- If $c e \rightarrow a b$ is an edge, then either we have the cycle $c e \rightarrow a b \rightarrow c d \rightarrow$ $b e \rightarrow a f \rightarrow c e$, or we have the shortcut with the shortcutting edge $c e \rightarrow a f$ and the long path $c e \rightarrow a b \rightarrow c d \rightarrow b e \rightarrow a f$. Thus, we must have $a b \rightarrow c e$.

- If $a f \rightarrow c e$ is an edge, then $a b \rightarrow c d \rightarrow b e \rightarrow a f \rightarrow c e$ is the long path and $a b \rightarrow c e$ is the shortcutting edge in a shortcut. Thus, we must have $c e \rightarrow a f$.

- Replacing ce by de in the last two bullet points, we see that $a b \rightarrow d e$ and $d e \rightarrow$ af are edges.

- If $c e \rightarrow d f$ is an edge, then $c e \rightarrow d f \rightarrow b e \rightarrow a f$ is the long path and $c e \rightarrow a f$ is the shortcutting edge in a shortcut. Thus, we must have $d f \rightarrow c e$. 
- From the graph induced by $d f$, be, af and $b c$, by Lemma 7, we must have $d f \rightarrow b c$ and $b c \rightarrow a f$.

- From the graph induced by $a b, d f$, de and $b c$, to avoid a shortcut, we must have $d e \rightarrow b c$.

- From the graph induced by $a b$, de, ef and $b c$, by Lemma 7 , we must have $a b \rightarrow$ ef and ef $\rightarrow b c$.

- From the graph induced by ef, $b c, b d$ and $a f$, by Lemma 7, we must have $e f \rightarrow b d$ and $b d \rightarrow a f$.

- From the graph induced by $a b, e f$, ce and $b d$, by Lemma 7 , we must have $a b \rightarrow c e$ and $c e \rightarrow b d$.

- From the graph induced by $d f$, $c e, a e$ and $b d$, by Lemma 7, we must have $d f \rightarrow a e$ and $a e \rightarrow b d$.

- From the graph induced by $c d$, ae, be and $d f$, we must have $c d \rightarrow a e$ to avoid a shortcut.

- From the graph induced by $a b, c f, c d$ and $a e$, we must have $c f \rightarrow a e$ to avoid a shortcut.

- From the graph induced by $c f, a e$, de and $b c$, we must have $a e \rightarrow b c$ to avoid a shortcut.

But we obtain a contradiction, since there is a shortcut with the long path $c d \rightarrow a e \rightarrow b c \rightarrow a f$ and the shortcutting edge $c d \rightarrow a f$. Thus, $K(6,2)$ is not semi-transitively orientable.

Remark 12. We note that $K(6,2)$, and thus any $K(m, 2)$ for $m \geq 6$, is not a minimal non-semi-transitive graph. Software check (using [5]) shows that removing any vertex in $K(6,2)$ gives a non-semi-transitive graph.

The following theorem generalizes Theorem [1].

Theorem 13. For $n \geq 15 k-24$ and $k \geq 2, K(n, k)$ is not semi-transitive.

Proof. We claim that such a $K(n, k)$ contains $K(6,2)$ as an induced subgraph, and thus is non-semi-transitive by Theorem 11, Indeed, consider inserting to each 2-subset involved in building $K(6,2)(k-2)$ distinct elements so that no two 2 -subsets receive the same new element. Then, the number of new elements is $15(k-2)$, and the total number of elements is 


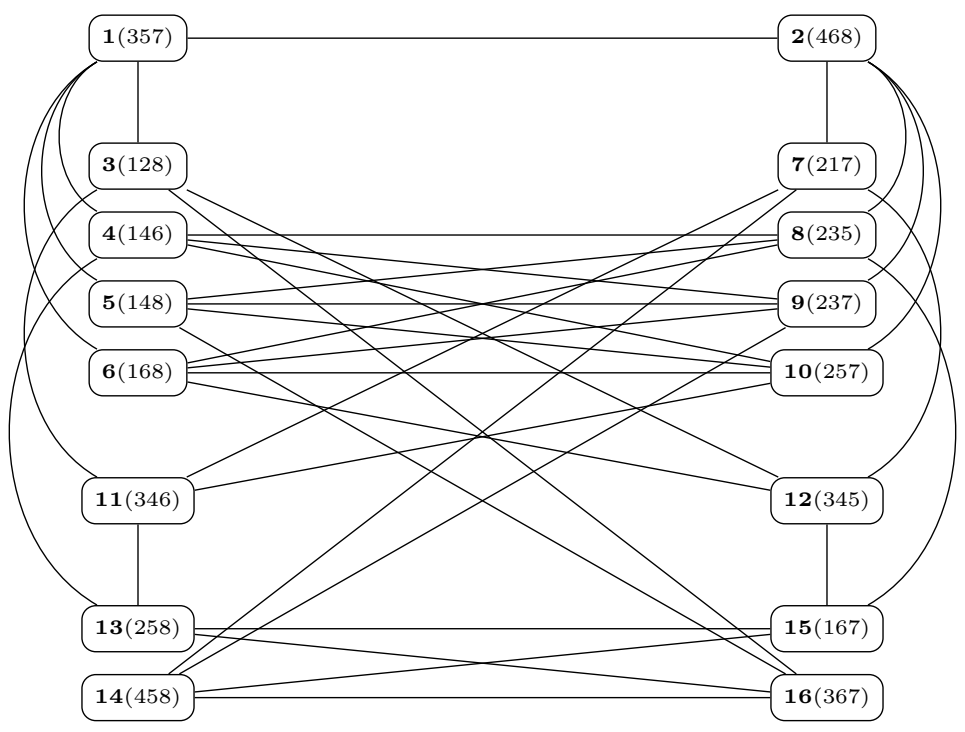

Figure 1: A minimal non-semi-transitive subgraph $S$ of $K(8,3)$. Name of a vertex is in bold, and the set partition corresponding to it is in parenthesis.

$6+15(k-2)=15 k-24$, where 6 came from the 6 elements used to build $K(6,2)$. Our construction shows that $K(6,2)$ is an induced subgraph in $K(15 k-24, k)$ since no edge in $K(6,2)$ is affected by the construction, and thus in any $K(n, k), n \geq 15 k-24$, by Lemma 10. Since $K(6,2)$ is non-semi-transitive by Theorem 11, $K(n, k)$ is non-semi-transitive for $n \geq 15 k-24$.

To extend our knowledge on semi-transitivity of Kneser graphs to the unknown cases, we have looked at $K(8,3)$ having 56 vertices and 280 edges. Using the software [5], we have learned that the subgraph of $K(8,3)$ formed by the 46 lexicographically smallest vertices $(123,124,126$, etc) is semitransitive, and a semi-transitive orientation was found within 2 seconds. However, adding one more vertex to the subgraph (456, the 47th lexicographically smallest one), resulted in no result returned by the software within a few hours, which was an indication, but not a given fact, that the graph may not be semi-transitive. Thus, our next goal was to find a non-semi-transitive subgraph $S$ of $K(8,3)$ of a smaller size, for which the software would return a definite answer on non-semi-transitivity of $S$, and thus of $K(8,3)$. Such a graph $S$, presented in Figure 1, was found using clustering nodes into independent sets and then eliminating certain nodes. 
Checking non-semi-transitivity of $S$ takes just below 4 seconds using the software [5], and the minimality of $S$ is straightforward to check using the same software. $S$ contains 16 vertices and 36 edges.

Of course, it would be desirable to find a non-computer based proof of non-semi-transitivity of $K(8,3)$, e.g. similar to that of non-semi-transitivity of $K(6,2)$ in Theorem 11, but we were not able to achieve it. We note that determining if a triangle-free graph is semi-transitive is an NP-hard problem [7], and presenting all $2^{35}$ orientations (one edge can be assumed to have any fixed orientation) and showing a shortcut, or cycle, in each of them is not feasible for a human. In fact, there is the branching method explained in Section 4.5 in [9] (also, see [3]) to dramatically decrease the number of cases to consider while proving that a graph is not semi-transitive. The basic idea of the method is to avoid branching for those edges for which orientation is uniquely determined in order to be semi-transitive. However, this method, being efficient for not so many edges, still leads to too many cases to consider for $S$, and thus is rather useless in the situation.

Remark 14. $S$ in Figure 1 is the first explicit example of a triangle-free non-semi-transitive graph. The existence of such graphs was established in [6] using Erdős' theorem (also see [9, Section 4.4]).

\section{Semi-transitivity of the complement of Kneser graphs}

Theorem 15. For $n \leq 2 k, \overline{K(n, k)}$ is semi-transitive.

Proof. Clearly, if $n<2 k$, then $\overline{K(n, k)}$ is a complete graph (no pair of $k$ subsets is disjoint), and thus it is semi-transitive. On the other hand, if $n=2 k$, then $\overline{K(n, k)}$ is a complete graph with a perfect matching removed (each non-edge is formed by a $k$-subset and its complement). Label $\overline{K(2 k, k)}$ so that the non-edges are formed by the vertices $2 i-1$ and $2 i$ for $1 \leq i \leq k$ and let $x=\left(\begin{array}{l}n \\ k\end{array}\right)$. Then, the word $1234 \cdots(x-1) x 2143 \cdots x(x-1)$ represents $\overline{K(2 k, k)}$, and by Theorem $[8, \overline{K(2 k, k)}$ is semi-transitive.

Lemma 16. For $k \geq 4$, we have $\left(\begin{array}{c}2 k \\ k-1\end{array}\right)+k<\frac{1}{2}\left(\begin{array}{c}2 k+1 \\ k\end{array}\right)-2$.

Proof. Using $\left(\begin{array}{c}2 k+1 \\ k\end{array}\right)=\left(\begin{array}{c}2 k \\ k\end{array}\right)+\left(\begin{array}{c}2 k \\ k-1\end{array}\right)$, we need to prove that

$$
\frac{1}{2}\left(\begin{array}{c}
2 k \\
k-1
\end{array}\right)+k<\frac{1}{2}\left(\begin{array}{c}
2 k \\
k
\end{array}\right)-2, \text { or }
$$




$$
\begin{gathered}
\frac{(2 k) !}{(k-1) !(k+1) !}+2 k<\frac{(2 k) !}{k ! k !}-4, \text { or } \\
k(2 k) !+2 k k !(k+1) !<(k+1)(2 k) !-4 k !(k+1) ! \text { or } \\
(2 k+4) k !(k+1) !<(2 k) !
\end{gathered}
$$

The last statement can be proved by induction on $k$ with the easy to check base case of $k=4$. Indeed, using the induction hypothesis, we have

$$
\begin{gathered}
(2(k+1)) !=(2 k+2)(2 k+1)(2 k) !>(2 k+2)(2 k+1)(2 k+4) k !(k+1) ! \\
>(2 k+6)(k+1) !(k+2) !
\end{gathered}
$$

where the last inequality follows from the easy to see, for $k \geq 1$, inequality

$$
(2 k+2)(2 k+1)(2 k+4)>(2 k+6)(k+2)(k+1),
$$

or $6 k^{3}+16 k^{2}+6 k-4>0$.

Theorem 17. For $k \geq 2$, the graph $\overline{K(2 k+1, k)}$ is not semi-transitive.

Proof. For $k=2$, we note that $\overline{K(5,2)}$ is the line graph of $K_{5}$, and it is proved in [10] to be non-word-representable, and thus, $\overline{K(5,2)}$ is not semitransitive by Theorem 8 .

Let $k=3$, and suppose that $\overline{K(7,3)}$ admits a semi-transitive orientation. Fix such an orientation. By Theorem 5 , the chromatic number of $\overline{K(7,3)}$ is 18 , and thus, by Theorem 1, $\overline{K(7,3)}$ contains a directed path $X_{1} \rightarrow X_{2} \rightarrow$ $\cdots \rightarrow X_{18}$. Moreover, by Theorem 4 , the largest clique in $\overline{K(7,3)}$ is of size 15, and thus if $X_{1} \rightarrow X_{i}$ is an edge for $i \in\{16,17,18\}, X_{1} \rightarrow X_{i}$ would be the shortcutting edge for the long path $X_{1} \rightarrow X_{2} \rightarrow \cdots \rightarrow X_{i}$ (the graph induced by $X_{1}, X_{2}, \ldots, X_{i}$ cannot be transitive as that would mean that $\overline{K(7,3)}$ has a clique of size $>15)$. Therefore, $X_{1}$ is not connected to $X_{16}, X_{17}$ and $X_{18}$, so that if $X_{1}=123$ (without loss of generality), then $X_{16}, X_{17}$ and $X_{18}$ are formed using the elements in $\{4,5,6,7\}$. Without loss of generality, assume that $X_{2}$ involves the element 4 . Since at least one of $X_{17}$ and $X_{18}$ must involve 4 , say $X_{m}$, where $m \in\{17,18\}, X_{2} \rightarrow X_{m}$ must be the shortcutting edge with the long path $X_{2} \rightarrow X_{3} \rightarrow \cdots \rightarrow X_{m}$. Contradiction. Thus, $\overline{K(7,3)}$ is not semi-transitive.

Finally, let $k \geq 4$ and suppose that $K(2 k+1, k)$ admits a semi-transitive orientation. Fix such an orientation. By Theorem 5 , the chromatic number of $\overline{K(2 k+1, k)}$ is $t=\left[\frac{1}{2}\left(\begin{array}{c}2 k+1 \\ k\end{array}\right)\right]$ and thus, by Theorem 1, $\overline{K(2 k+1, k)}$ contains a directed path $X_{1} \rightarrow X_{2} \rightarrow \cdots \rightarrow X_{t}$. Moreover, by Theorem 4 , 
the largest clique in $\overline{K(2 k+1, k)}$ is of size $s=\left(\begin{array}{c}2 k \\ k-1\end{array}\right)$. Thus, $X_{1}$ cannot be connected to $X_{i}$ for $s+1 \leq i \leq t$, and the number of such $X_{i}$ s cannot exceed $\left(\begin{array}{c}(2 k+1)-k \\ k\end{array}\right)=k+1$ (the $\bar{k}$ elements used in $X_{1}$ are not available for $X_{i} \mathrm{~s}$ ). But then, we must have the following inequality

$$
\begin{gathered}
t-(s+1)+1 \leq k+1 \Rightarrow\left[\frac{1}{2}\left(\begin{array}{c}
2 k+1 \\
k
\end{array}\right)\right]-\left(\begin{array}{c}
2 k \\
k-1
\end{array}\right) \leq k+1 \Rightarrow \\
\frac{1}{2}\left(\begin{array}{c}
2 k+1 \\
k
\end{array}\right)-1-\left(\begin{array}{c}
2 k \\
k-1
\end{array}\right) \leq k+1,
\end{gathered}
$$

which contradicts Lemma 16. Thus, $K(2 k+1, k)$ is not semi-transitively orientable for $k \geq 4$.

As an immediate corollary to Theorems 15 and 17 and Lemma 10, we have the following result.

Theorem 18. The complement graph $\overline{K(n, k)}$ of $K(n, k)$ is semi-transitive if and only if $n \leq 2 k$.

Proof. By Theorem [15, $\overline{K(n, k)}$ is semi-transitive if $n \leq 2 k$. Now, suppose that $n>2 k$. Since $\overline{K(2 k+1, k)}$ is an induced subgraph in $\overline{K(n, k)}$ by Lemma 10, and $\overline{K(2 k+1, k)}$ is non-semi-transitive by Theorem 17, $\overline{K(n, k)}$ is also non-semi-transitive.

\section{Concluding remarks}

In this paper, we show that for $n \geq 15 k-24, K(n, k)$ is not semi-transitive, while for $k \leq n \leq 2 k+1, K(n, k)$ is semi-transitive. Also, we have used computations to show that the triangle-free graph $K(8,3)$ is not semi-transitive. Moreover, we have completely characterized semi-transitivity of the complement graph $\overline{K(n, k)}$ by showing that $\overline{K(n, k)}$ is semi-transitive if and only if $n \geq 2 k$. We conclude the paper with the following open problems.

- Give a non-computer based proof of non-semi-transitivity of $K(8,3)$.

- More generally, is $K(2 k+2, k)$ non-semi-transitive for any $k \geq 3$ ? If that would be the case, then we would complete the classification of semi-transitive Kneser graphs by Lemma 10 and Theorems 11 and 13 . namely, that would imply that a Kneser graph is semi-transitive if and only if it is 3 -colourable. 
- Are there any smaller triangle-free non-semi-transitive graphs (by the number of vertices and/or the number of edges) than the graph $S$ in Figure 1]? Possible candidates for such a graph could be some subgraphs of $K(8,3)$, which could then also help to prove rigorously nonsemi-transitivity of $K(8,3)$ (the fewer than 36 edges in such a graph could possibly be handled by the branching method in [9, Section 4.5]). As a relevant observation to searching for candidates here, note that triangle-free planar graphs are always semi-transitive as they are 3-colorable [6].

\section{Acknowledgment}

We are grateful to Li-Da Tong for raising our interest in Kneser graphs.

\section{References}

[1] P. Akrobotu, S. Kitaev, Z. Masarova. On word-representability of polyomino triangulations, Siberian Adv. in Math. 25 (2015) 1, 1-10.

[2] Zs. Baranyai. On the factorization of the complete uniform hypergraph, in: "Infinite and Finite Sets" (Proc. Intern. Coll. Keszthely, 1973; A. Hajnal, R. Rado and V. T. Sós, eds.), Bolyai J. Mat. Társulat, Budapest \& North-Holland, Amsterdam, 1975, pp. 91-108.

[3] A. Collins, S. Kitaev, V. Lozin. New results on word-representable graphs. Discr. Appl. Math. 216 (2017) 136-141.

[4] P. Erdős, C. Ko, R. Rado. Intersection theorems for systems of finite sets, Quarterly Journal of Mathematics. Oxford. Second Series 12 (1961) 313-320.

[5] M. Glen. Software available at personal.cis.strath.ac.uk/ sergey.kitaev/word-representable-graphs.html

[6] M. M. Halldórsson, S. Kitaev, A. Pyatkin. Alternation graphs, Lect. Notes in Comp. Sci. 6986 (2011) 191-202. Proceedings of the 37th International Workshop on Graph-Theoretic Concepts in Computer Science, WG 2011, Tepla Monastery, Czech Republic, June 21-24, 2011.

[7] M. M. Halldórsson, S. Kitaev, A. Pyatkin. Semi-transitive orientations and word-representable graphs, Discr. Appl. Math. 201 (2016) 164-171. 
[8] S. Kitaev. A Comprehensive Introduction to the Theory of WordRepresentable Graphs. Lect. Notes in Comp. Sci. 10396 (2017) 36-67.

[9] S. Kitaev, V. Lozin. Words and Graphs, Springer, 2015.

[10] S. Kitaev, P. Salimov, C. Severs, H. Ulfarsson. Word-representability of line graphs, Open J. Discr. Math. 1 (2011) 2, 96-101.

[11] S. Kitaev, S. Seif. Word problem of the Perkins semigroup via directed acyclic graphs. Order 25 (2008) 3, 177-194.

[12] L. Lovász. Kneser's conjecture, chromatic number, and homotopy, $J$. Combin. Theory, Series A 25(3) (1978) 319-324.

[13] T. Mütze, J. Nummenpalo, B. Walczak. Sparse Kneser graphs are Hamiltonian, STOC 2018 Proceedings of the 50th Annual ACM SIGACT Symposium on Theory of Computing (2018) 912-919. 